\title{
Effects of Plantar Flexor Muscle Static Stretching Alone and Combined With Massage on Postural Balance
}

\author{
Ladan Hemmati, PhD, Zahra Rojhani-Shirazi, PhD, Samaneh Ebrahimi, PhD \\ Department of Physiotherapy, Shiraz University of Medical Sciences, Shiraz, Iran
}

\begin{abstract}
Objective To evaluate and compare the effects of stretching and combined therapy (stretching and massage) on postural balance in people aged 50 to 65 years.

Methods Twenty-three subjects participated in this nonrandomized clinical trial study. Each participant randomly received plantar flexor muscle stretching ( 3 cycles of 45 seconds with a 30 -second recovery period between cycles) alone and in combination with deep stroking massage (an interval of at least 30 minutes separated the two interventions). The data were recorded with a force platform immediately after each condition with eyes open and closed. The center of pressure displacement and velocity along the mediolateral and anteroposterior axes were calculated under each condition. The data were analyzed with multiple-pair t-tests.

Results The center of pressure displacement and velocity along the mediolateral axis increased after both stretching and the combined intervention. There were significant differences in both values between participants in the stretching and combined interventions $(\mathrm{p}<0.05)$.

Conclusion Plantar flexor muscle stretching (for 45 seconds) combined with deep stroking massage may have more detrimental effects on postural balance than stretching alone because each intervention can intensify the effects of the other.
\end{abstract}

Keywords Postural balance, Muscle stretching exercises, Massage

\section{INTRODUCTION}

In recent years, muscle stretching has been widely used in rehabilitation and performance enhancement to increase flexibility [1] and pain-free range of motion
(ROM) [2] and to decrease the risk of injury [3]. Despite the benefits of stretching, recent studies have reported an adverse effect on muscle strength and performance when static stretching was used. For example, it has been reported that static stretching may decrease force

Received August 31, 2015; Accepted January 21, 2016

Corresponding author: Zahra Rojhani-Shirazi

Department of Physiotherapy, School of Rehabilitation Sciences, Shiraz University of Medical Sciences, Abiverdi 1 Avenue, Chamran Boulevard, Shiraz, Iran. Tel: +98-713-6261081, Fax: +98-713-6272495, E-mail: rojhaniz@sums.ac.ir

ORCID: Ladan Hemmati (http://orcid.org/0000-0003-4618-8603); Zahra Rojhani-Shirazi (http://orcid.org/0000-0003-2878-9560); Samaneh Ebrahimi (http://orcid.org/0000-0001-9330-3104).

(c) This is an open-access article distributed under the terms of the Creative Commons Attribution Non-Commercial License (http://creativecommons.org/ licenses/by-nc/4.0) which permits unrestricted noncommercial use, distribution, and reproduction in any medium, provided the original work is properly cited. Copyright (c) 2016 by Korean Academy of Rehabilitation Medicine 
production $[4,5]$ and power outputs $[6,7]$. This reduced muscle force has been attributed to changes in musculotendinous stiffness $[4,8]$ and impaired neural output $[5,9]$. Therefore, these alterations in muscle force production may affect postural balance response because balance requires muscle force to maintain the center of gravity within the base of support [10]. A number of studies have found that static stretching can decrease balance [11,12], and reduced balance may be predicted to increase the risk of falling, thus affecting quality of life in that balance is a prerequisite for activities of daily living [13].

Falling is a serious problem, particularly in middle aged and older adult populations [14]; in the United States, falls are the main cause of injury-related hospital admissions and death associated with aging [14]. The aging process results in reduced afferent sensory information, which is necessary for producing muscle force and maintaining postural balance. Therefore, improving sensory information can help in maintaining upright posture and can also prevent falling [15]. For example, Vaillant et al. [15] reported that massage can improve postural balance by increasing somatosensory information in elderly adults. However, other evidence suggests that massage causes a decline in muscle force [15]. In this connection, Goodwin [16] demonstrated that massage can decrease vertical jump performance and suggested that this result could be attributed to reduced muscle stiffness and impaired neural output.

The results available so far regarding the effects of stretching on postural balance raise the question of whether or not the use of a therapeutic intervention after stretching can modify balance parameters. Thus, the aims of this study were first to evaluate the effects of stretching and a combined intervention (stretching and massage) on postural balance with eyes closed and open in people aged 50 to 65 years and second to compare the effects of these two interventions with each other on postural control.

\section{MATERIALS AND METHODS}

\section{Participants}

Twenty-three adults aged 50 to 65 years participated in this quasi-experimental study through nonrandomized sampling; written informed consent was obtained from each patient prior to his or her participation. The study was approved by the Research Ethics Committee of Shiraz University of Medical Sciences. The exclusion criteria were low back pain, alcohol intake within 24 hours of testing, history of surgery in the lower extremities, vestibular dysfunction (with physician diagnosis) or balance disorder (such as ankle instability), and history of neurological disorder.

\section{Procedure}

In order to measure postural balance, the participants stood barefoot on a force platform (Kistler Instrument, Winterthur, Switzerland) set at a sample rate of $100 \mathrm{~Hz}$ in a neutral position (feet abducted at $10^{\circ}$, heels separated by $10 \mathrm{~cm}$ ), and they were asked to sway as little as possible. The tests were done in three conditions (no intervention, stretching, and combined intervention) with eyes open and closed on the force platform. Each test (30-second duration) was repeated three times with 10 -second rests between the trials.

The participants received both static stretching alone and the combined intervention (stretching and deep stroking massage) in random order on the same day. In order to stretch the plantar flexor muscles of both lower extremities, the participants were asked to sit on a specially built device that caused static dorsiflexion of about $20^{\circ}$ (Fig. 1). The stretching position was applied to the participant's discomfort threshold and maintained for 45 seconds. It was repeated three times, with a 30 -second recovery period between cycles.

In the combined intervention protocol, both muscle stretching and deep stroking massage were used for each participant in random order. The massage involved slow, rhythmic, deep stroking of both plantar flexor muscles

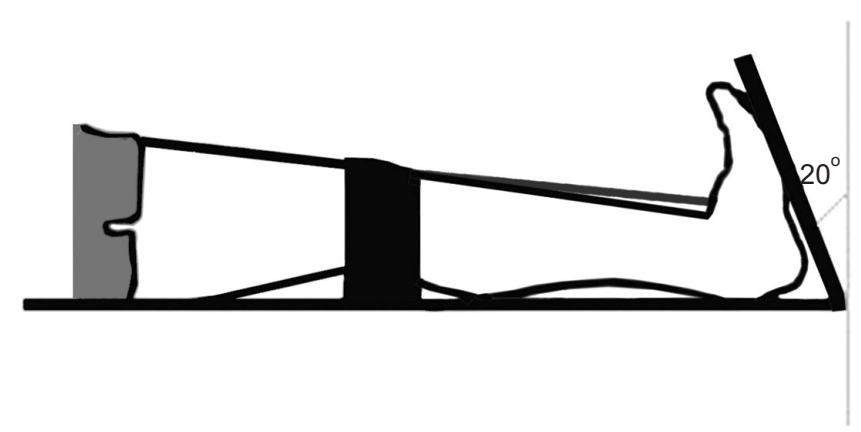

Fig. 1. Schematic diagram of the device and position used for the stretching. 
for 8 minutes while the participant was lying prone on a standard treatment table. An expert physiotherapist performed the massage with oil for all participants. To avoid carry-over effects, an interval of at least 30 minutes separated every two interventions; this duration was selected based on the results of a pilot study.

\section{Data analysis}

The raw data were exported to Visual 3D software (CMotion Inc., Rockville, MD, USA) and filtered using a fourth-order Butterworth low-pass filter with a cut-off frequency of $12 \mathrm{~Hz}$; 30 seconds of data were recorded for each test. The center of pressure (COP) displacement and velocity along the mediolateral and anteroposterior axes were calculated to evaluate postural balance parameters.

\section{Statistical analysis}

The Statistical Package for the Social Sciences ver. 16.0 (SPSS Inc., Chicago, IL, USA) was used to analyze the data. Data normality was tested with the KolmogorovSmirnov test ( $p>0.05)$, and multiple-pair t-tests were used for statistical evaluation. Significance was set at $\mathrm{p}<0.05$.

\section{RESULTS}

The results of this study demonstrated that there was a significant difference in mean mediolateral COP displacement and velocity after static stretching and the

Table 1. Mean COP displacement and velocity between no intervention and the stretching intervention with eyes closed and open

\begin{tabular}{|c|c|c|c|c|c|c|}
\hline \multirow{2}{*}{\multicolumn{3}{|c|}{ Variable }} & \multicolumn{2}{|c|}{ Eye open } & \multicolumn{2}{|c|}{ Eye close } \\
\hline & & & \multirow{2}{*}{$\begin{array}{c}\text { Mean } \pm \text { SD } \\
2.516 \pm 1.334\end{array}$} & \multirow{2}{*}{$\begin{array}{c}\text { p-value } \\
0.30\end{array}$} & \multirow{2}{*}{$\begin{array}{l}\text { Mean } \pm \text { SD } \\
2.370 \pm 0.597\end{array}$} & \multirow{2}{*}{$\begin{array}{l}\text { p-value } \\
<0.001^{*}\end{array}$} \\
\hline COP displacement (mm) & ML & No intervention & & & & \\
\hline & & Stretching & $2.304 \pm 0.730$ & & $2.813 \pm 0.940$ & \\
\hline & $\mathrm{AP}$ & No intervention & $2.029 \pm 0.763$ & 0.89 & $2.148 \pm 0.841$ & 0.32 \\
\hline & & Stretching & $2.013 \pm 0.573$ & & $2.316 \pm 0.751$ & \\
\hline \multirow[t]{4}{*}{ COP velocity (mm/s) } & ML & No intervention & $0.120 \pm 0.056$ & 0.51 & $0.124 \pm 0.040$ & $<0.001^{*}$ \\
\hline & & Stretching & $0.115 \pm 0.037$ & & $0.142 \pm 0.047$ & \\
\hline & $\mathrm{AP}$ & No intervention & $0.111 \pm 0.061$ & 0.57 & $0.113 \pm 0.040$ & 0.68 \\
\hline & & Stretching & $0.106 \pm 0.039$ & & $0.116 \pm 0.037$ & \\
\hline
\end{tabular}

$\mathrm{COP}$, center of pressure; ML, mediolateral; AP, anteroposterior.

${ }^{*} \mathrm{p}<0.05$.

Table 2. Mean COP displacement and velocity between no intervention and the combined intervention with eyes closed and open

\begin{tabular}{|c|c|c|c|c|c|c|}
\hline \multirow{2}{*}{\multicolumn{3}{|c|}{ Variable }} & \multicolumn{2}{|c|}{ Eye open } & \multicolumn{2}{|c|}{ Eye close } \\
\hline & & & \multirow{2}{*}{$\begin{array}{c}\text { Mean } \pm \text { SD } \\
2.516 \pm 1.334\end{array}$} & \multirow{2}{*}{$\begin{array}{c}\text { p-value } \\
0.12\end{array}$} & \multirow{2}{*}{$\begin{array}{c}\text { Mean } \pm \text { SD } \\
2.370 \pm 0.597\end{array}$} & \multirow{2}{*}{$\frac{p \text {-value }}{<0.001^{*}}$} \\
\hline COP displacement $(\mathrm{mm})$ & ML & No intervention & & & & \\
\hline & & Combined & $2.868 \pm 1.665$ & & $3.307 \pm 1.229$ & \\
\hline & $\mathrm{AP}$ & No intervention & $2.029 \pm 0.763$ & 0.77 & $2.148 \pm 0.841$ & 0.24 \\
\hline & & Combined & $2.075 \pm 0.749$ & & $2.306 \pm 0.601$ & \\
\hline \multirow[t]{4}{*}{ COP velocity $(\mathrm{mm} / \mathrm{s})$} & ML & No intervention & $0.120 \pm 0.056$ & 0.20 & $0.124 \pm 0.040$ & $<0.001^{*}$ \\
\hline & & Combined & $0.134 \pm 0.058$ & & $0.169 \pm 0.061$ & \\
\hline & AP & No intervention & $0.111 \pm 0.061$ & 0.96 & $0.113 \pm 0.040$ & 0.84 \\
\hline & & Combined & $0.111 \pm 0.045$ & & $0.114 \pm 0.042$ & \\
\hline
\end{tabular}

$\mathrm{COP}$, center of pressure; ML, mediolateral; $\mathrm{AP}$, anteroposterior.

${ }^{*} \mathrm{p}<0.05$. 
Table 3. Mean differences in COP displacement and velocity between stretching and the combined intervention with eyes closed and open

\begin{tabular}{|c|c|c|c|c|c|c|}
\hline \multirow{2}{*}{\multicolumn{3}{|c|}{ Variable }} & \multicolumn{2}{|c|}{ Eye open } & \multicolumn{2}{|c|}{ Eye close } \\
\hline & & & \multirow{2}{*}{$\begin{array}{c}\text { Mean } \pm \text { SD } \\
2.868 \pm 1.665\end{array}$} & \multirow{2}{*}{$\begin{array}{c}\text { p-value } \\
0.05\end{array}$} & \multirow{2}{*}{$\begin{array}{r}\text { Mean } \pm \text { SD } \\
3.307 \pm 1.229\end{array}$} & \multirow{2}{*}{$\begin{array}{l}\text { p-value } \\
<0.001^{*}\end{array}$} \\
\hline COP displacement (mm) & ML & Combined & & & & \\
\hline & & Stretching & $2.304 \pm 0.730$ & & $2.813 \pm 0.940$ & \\
\hline & $\mathrm{AP}$ & Combined & $2.075 \pm 0.749$ & 0.72 & $2.306 \pm 0.601$ & 0.99 \\
\hline & & Stretching & $2.013 \pm 0.573$ & & $2.316 \pm 0.751$ & \\
\hline \multirow[t]{4}{*}{ COP velocity (mm/s) } & ML & Combined & $0.134 \pm 0.058$ & $0.03^{*}$ & $0.169 \pm 0.061$ & $<0.001^{*}$ \\
\hline & & Stretching & $0.115 \pm 0.037$ & & $0.142 \pm 0.047$ & \\
\hline & $\mathrm{AP}$ & Combined & $0.111 \pm 0.045$ & 0.39 & $0.114 \pm 0.042$ & 0.83 \\
\hline & & Stretching & $0.106 \pm 0.039$ & & $0.116 \pm 0.037$ & \\
\hline
\end{tabular}

$\mathrm{COP}$, center of pressure; ML, mediolateral; AP, anteroposterior.

${ }^{*} \mathrm{p}<0.05$.

combined intervention when participants were tested with their eyes closed (Tables 1, 2). In other words, mediolateral COP displacement and velocity increased after both stretching and the combined interventions. Moreover, the displacement and velocity were greater after the combined intervention than after stretching alone (Table 3 ).

\section{DISCUSSION}

The results showed that mediolateral postural sway increased significantly after stretching when participants were tested with their eyes closed. This was consistent with earlier findings by Nagano et al. [12], who evaluated the effects of plantar flexor muscle stretching for $3 \mathrm{~min}$ utes on postural sway during quiet standing; participants stood barefoot on a force plate and were asked to sway as little as possible, and the results showed that static stretching of the plantar flexor muscles can increase postural sway. Behm et al. [11] investigated the effects of static stretching of the quadriceps, hamstrings, and plantar flexors on balance control. This study's subjects were tested with a computerized wobble board before and after static stretching, and the results showed that balance control was impaired after an acute bout of static stretching. The findings of these two studies demonstrate that stretching has an adverse effect on balance.

In this connection, a number of studies have noted that passive stretching may inhibit normal performance by reducing muscle power output and muscle force [4-7]. Two primary hypotheses have been proposed to explain these decreases: mechanical factors such as changes in muscle stiffness and neural factors such as decreased motor unit activation or firing frequency or altered reflex sensitivity $[8,17]$.

Passive stretching induces decreased musculotendinous unit (MTU) stiffness and increased MTU length, and these alterations may affect discharges from the muscle spindle and Golgi tendon organ (GTO) to maintain postural balance. Detecting and monitoring muscle tension with the GTO may be delayed because of the increased electromechanical delay in the series elastic component of force production. In addition, reduced excitatory drive from the Ia afferents due to increased MTU compliance can decrease excitation of the motor neuron pool and thus affect postural balance $[5,12]$.

We found a significant difference in mean mediolateral COP displacement and velocity after the combined intervention when participants were tested with their eyes closed; in addition, the displacement and velocity differed significantly between the stretching alone and the combined intervention conditions. Deep stroking massage appeared to have a negative impact on postural balance because the combined intervention had more adverse effects on balance than did stretching alone. This finding is inconsistent with the results of previous studies, according to which friction massage and mobilization of the feet and ankles improved postural balance by stimulating local skin receptors and increasing somatosensory information $[15,18]$. The probable cause of the discrepancies between studies lies in the different types of massage used and the magnitudes of contact pressure.

A number of studies suggest that massage reduces mus- 
cle stiffness and lengthens the massaged muscles [19], and according to the length-tension relationship, when the skeletal muscle is lengthened, the number of actin/ myosin cross-bridges declines, resulting in reduced force output without corresponding reduced neural activation [19]. Our results suggest that the effect of massage is similar to that of stretching. Accordingly, the combination of massage with stretching may result in greater muscle lengthening than stretching alone, leading to a greater loss of muscle force and consequently greater impairment in postural balance [19].

Although stretching was applied in the anteroposterior direction, the changes in postural balance parameters were not statistically significant in this plane. This finding can be attributed to the age-related reduction in dorsiflexion ROM. It has been stated that the aging process results in reduced ROM in all joints and that dorsiflexion ROM undergoes the greatest decrease during aging [20]. The human body uses various strategies to control balance. The body is a closed biomechanical chain, so both its proximal and distal parts help to fine-tune the location of the center of mass in the base of support [21]. If ankle ROM is decreased, other joints must compensate for the alteration to avoid motion deviations [21]. Decreased ankle dorsiflexion ROM appears to be accompanied by greater knee motion in the frontal plane [22]. Therefore, it can be considered that the increased sway in the mediolateral direction may be due to a compensatory mechanism in a closed kinetic chain of the human body in response to decreased ankle ROM and fatigue in the sagittal plane.

In contrast, the greater mediolateral postural sway compared with anteroposterior sway could be attributed to probable gluteus medius muscle weakness that occurs with aging. Serratrice et al. [23] reported progressive muscular weakening in the pelvic and shoulder girdles with less distal muscle involvement in older adults; these authors noted that symptoms of myopathic disease could be seen in the proximal muscles with aging. In the current experiment, we did not measure gluteus medius muscle force, and this is a potential limitation of our study.

The increase in postural sway when the participants kept their eyes closed was statistically significant. This finding is consistent with the well-documented phenomenon of improved balance control with visual input [24].
It has been stated that postural balance involves multiple sensory systems: visual, vestibular and somatosensory [13]. Other studies have also confirmed vision as an important part of postural balance [12]; therefore, it is unsurprising that balance was impaired when the participants' vision was removed.

A number of limitations in the present study deserve mention. Firstly, we did not measure pelvic girdle muscle strength. Secondly, we measured the immediate effects of one session of therapeutic intervention on postural balance, and the immediate influence of a single intervention should not be compared with the long-term effects of prolonged interventions. Therefore, additional well-designed controlled trials with a larger number of participants are needed to judge whether or not the interventions we compared compromise postural stability. Thirdly, we did not evaluate the clinical relevance of the results.

In conclusion, plantar flexor muscle stretching (for 45 seconds) combined with deep stroking massage may have more detrimental effects on postural balance than stretching alone because each can intensify the effects of the other. Therefore, these therapeutic interventions can be a probable risk factor for falling during the aging process.

\section{CONFLICT OF INTEREST}

No potential conflict of interest relevant to this article was reported.

\section{ACKNOWLEDGMENTS}

This article was extracted from the MSc thesis written by Ladan Hemmati and supported by Shiraz University of Medical Sciences (Proposal No. 5939). Hereby, the authors would like to thank the participants for contributing their time and support to this study. The authors are also grateful for Dr. N. Shokrpour for improving the English in the manuscript.

\section{REFERENCES}

1. Ferber R, Osternig L, Gravelle D. Effect of PNF stretch techniques on knee flexor muscle EMG activity in older adults. J Electromyogr Kinesiol 2002;12:391-7. 
2. Harvey LA, Herbert RD. Muscle stretching for treatment and prevention of contracture in people with spinal cord injury. Spinal Cord 2002;40:1-9.

3. Wilson GJ, Wood GA, Elliott BC. The relationship between stiffness of the musculature and static flexibility: an alternative explanation for the occurrence of muscular injury. Int J Sports Med 1991;12:403-7.

4. Fowles JR, Sale DG, MacDougall JD. Reduced strength after passive stretch of the human plantarflexors. J Appl Physiol (1985) 2000;89:1179-88.

5. Behm DG, Button DC, Butt JC. Factors affecting force loss with prolonged stretching. Can J Appl Physiol 2001;26:261-72.

6. Young W, Elliott S. Acute effects of static stretching, proprioceptive neuromuscular facilitation stretching, and maximum voluntary contractions on explosive force production and jumping performance. Res Q Exerc Sport 2001;72:273-9.

7. Cornwell A, Nelson AG, Sidaway B. Acute effects of stretching on the neuromechanical properties of the triceps surae muscle complex. Eur J Appl Physiol 2002;86:428-34.

8. Taylor DC, Dalton JD Jr, Seaber AV, Garrett WE Jr. Viscoelastic properties of muscle-tendon unit: the biomechanical effects of stretching. Am J Sports Med 1990;18:300-9.

9. Avela J, Kyrolainen H, Komi PV. Altered reflex sensitivity after repeated and prolonged passive muscle stretching. J Appl Physiol (1985) 1999;86:1283-91.

10. Khanna P, Kapoor G, Zutshi K. Balance deficits and recovery timeline after different fatigue protocols. Indian J Physiother Occup Ther 2008;2:42-54.

11. Behm DG, Bambury A, Cahill F, Power K. Effect of acute static stretching on force, balance, reaction time, and movement time. Med Sci Sports Exerc 2004;36:1397-402.

12. Nagano A, Yoshioka S, Hay DC, Himeno R, Fukashiro S. Influence of vision and static stretch of the calf muscles on postural sway during quiet standing. Hum Mov Sci 2006;25:422-34.

13. Shirazi ZR, Jahromi FN. Comparison of the effect of selected muscle groups fatigue on postural control during bipedal stance in healthy young women. Niger Med J 2013;54:306-9.
14. Holt KR, Haavik H, Elley CR. The effects of manual therapy on balance and falls: a systematic review. J Manipulative Physiol Ther 2012;35:227-34.

15. Vaillant J, Rouland A, Martigne P, Braujou R, Nissen MJ, Caillat-Miousse JL, et al. Massage and mobilization of the feet and ankles in elderly adults: effect on clinical balance performance. Man Ther 2009;14:6614.

16. Goodwin J. A comparison of massage and sub-maximal exercise as warm-up protocols combined with a stretch for vertical jump performance. J Sports Sci 2002;20:48-9.

17. Zhao H, Wu YN, Hwang M, Ren Y, Gao F, GaeblerSpira D, et al. Changes of calf muscle-tendon biomechanical properties induced by passive-stretching and active-movement training in children with cerebral palsy. J Appl Physiol (1985) 2011;111:435-42.

18. Vaillant J, Vuillerme N, Janvey A, Louis F, Braujou R, Juvin R, et al. Effect of manipulation of the feet and ankles on postural control in elderly adults. Brain Res Bull 2008;75:18-22.

19. Hunter AM, Watt JM, Watt V, Galloway SD. Effect of lower limb massage on electromyography and force production of the knee extensors. Br J Sports Med 2006;40:114-8.

20. James B, Parker AW. Active and passive mobility of lower limb joints in elderly men and women. Am J Phys Med Rehabil 1989;68:162-7.

21. Friel K, McLean N, Myers C, Caceres M. Ipsilateral hip abductor weakness after inversion ankle sprain. J Athl Train 2006;41:74-8.

22. Padua DA, Clark MA. Knee injury prevention: hip and ankle strategies [Internet]. Albany: Lower Extremity Review Magazine; 2014 [cited 2016 Jul 15]. Available from: http://lermagazine.com/article/knee-injuryprevention-hip-and-ankle-strategies.

23. Serratrice G, Roux H, Aquaron R. Proximal muscular weakness in elderly subjects: report of 12 cases. J Neurol Sci 1968;7:275-99.

24. Shumway-Cook A, Woollacott MH. Motor control: translating research into clinical practice. 3rd ed. Philadelphia: Lippincott Williams \& Wilkins; 2007. p. 158-62. 